\title{
INCIDENCIA DEL DINERO ELECTRÓNICO EN EL PROCESO DE LAS ACTIVIDADES ECONÓMICAS.
}

Adrián Valencia Medranda, Libi Carol Caamaño, Lenin Balseca Tapia, Nathyn Collins Ventura, Isauro Domo Mendoza, Nury Vera Campuzano, José Villao Viteri, Lilia Valencia Cruzaty, Verónica Benavides Benítez, Paola Cortez Clavijo 


\title{
INCIDENCIA DEL DINERO ELECTRÓNICO EN EL PROCESO DE LAS ACTIVIDADES ECONÓMICAS.
}

\author{
Adrián Valencia Medranda ${ }^{1}$, Libi Carol Caamaño ${ }^{1}$, Lenin Balseca Tapia ${ }^{1}$, Nathyn Collins Ventura ${ }^{1}$, Isauro Domo \\ Mendoza $^{1}$, Nury Vera Campuzano ${ }^{1}$, José Villao Viteri ${ }^{1}$, Lilia Valencia Cruzaty ${ }^{1}$, Verónica Benavides Benítez ${ }^{1}$, Paola \\ Cortez Clavijo ${ }^{1}$. \\ ${ }^{1}$ Universidad Estatal Península de Santa Elena. Carrera de Contabilidad y Auditoria \\ Campus La Libertad, vía principal Santa Elena - La Libertad - Ecuador \\ E-mail: joseavm68@gmail.com
}

\section{Resumen}

El nuevo sistema de dinero electrónico es una tendencia innovadora del sistema monetario que favorece las políticas bancarias que tienen como objetivo suministrar los medios de pago necesarios para que el sistema económico opere con eficiencia, por eso es de mayor importancia analizar la incidencia de este, frente al procesos de la actividades económicas en las diferentes áreas comerciales, a partir de dos técnicas de estudio utilizadas, las encuestas realizadas a la población del cantón La Libertad provincia de Santa Elena, y las entrevistas de profundidad realizadas a los representantes de los entes de acuerdo a las inversiones realizadas por el Econ. Mateo Villalba, gerente del Banco Central del Ecuador y el Econ. César Robalino Gonzaga, director de la Asociación de Bancos Privados del Ecuador. En base al estudio, obtuvimos como resultados que cuando entre en vigencia este sistema monetario en las actividades económicas, incidirá en los siguientes procesos, con un 33\% en la vida cotidiana de las personas, debido al cambio, seguido con un $20 \%$ se menciona dos procesos; incidiendo de manera positiva que se agilizaran las transacciones comerciales y de manera negativa afectaría la economía familiar.

Palabras Claves: Dinero electrónico, incidencia, economía, procesos.

\begin{abstract}
The new electronic money system is an innovative trend of the monetary management, that favors banking policies that are intended to provide the means a payment way; necessary for the economic system for efficient operations, so it is more important to analyze the impact of this, comparing the processes of economic activities in different business areas, from two study techniques used, surveys the population of La Libertad province of Santa Elena, and in-depth interviews conducted to two important entities of the economy the economist Mateo Villalba, manager of the Central Bank of Ecuador \& the economist César Robalino Gonzaga, director of the Association of Private Banks of Ecuador. Based on the study, we obtained as results that the effect in monetary system of economic activities, will affect the following processes; with a $33 \%$ in the daily lives of people, because of the change, along with $20 \%$ in two mentioned processes: impacting positively the commercial transactions and affect negatively the family finances.
\end{abstract}

Keywords: Electronic money, incidence, economy, process. 


\section{Introducción}

El dinero electrónico es una nueva herramienta donde se realizan pagos, transferencias y compras -en moneda fraccionaria- utilizado a través de dispositivos electrónicos sin la necesidad de tener internet ni una cuenta en una entidad financiera. (Mateo Villalba, 2014).

El dinero electrónico tiene beneficios, ya que permitirá simplificar los pagos en moneda fraccionaria mediante el uso de dispositivos electrónicos, su seguridad está garantizada por el Banco Central del Estado que es el ente regulador. Por lo tanto, el presente artículo describe el potencial que tiene el sistema de dinero electrónico dentro de nuestro país, medido a través de un estudio de mercado realizado en el sector comercial del cantón La Libertad.

\subsection{Problemas derivados del desarrollo del dinero electrónico.}

El desarrollo de este sistema se establece dentro del entorno sistemático que conlleva a la economía del país, que a la vez influyen en el beneficio económico y social, dando soluciones en el entorno de sus operaciones mercantiles, por otra parte existirá una resistencia al cambio que mantiene la contraparte donde se enmarcan inconvenientes, que se relacionan al poco conocimiento que mantiene la población y que para ellos se demuestra al realizar una operación comercial, además de la protección al consumidor, que no son de interés de los bancos, porque se habla de la inseguridad de los dispositivos por las funciones que tiene el software que este maneja.

\subsection{Importancia del dinero electrónico.}

El dinero electrónico permitirá simplificar los pagos en moneda financiera mediante el uso de la telefonía, realizar pagos y transferencias de formas más rápida, segura, higiénica y barata.

Es más eficiente, no se deteriora y permite pagar el precio exacto, no tendrá cobro de las altas comisiones, porque será un servicio público sin fines de lucro.

Por otro lado se podrá pagar servicios públicos como el SRI, Registro Civil, luz, agua y teléfono, en tiendas autorizadas, supermercados, farmacias entre otros, y ofrecerá mayor cobertura en zonas alejadas que no cuentan con entidades financieras.

\section{Materiales y métodos.}

La investigación se llevó a cabo mediante una encuesta para conocer las ideas principales de la aplicación del sistema de dinero electrónico y lograr los objetivos planteados, se obtuvo una población de 100, mediante un muestreo aleatorio simple, con la finalidad de que todas las personas tengan la misma probabilidad de ser encuestadas (Bernal, 2010), en el cantón La Libertad, provincia de Santa Elena.

En las entrevistas realizadas a los directivos del Banco Central del Ecuador, se identificó el alcance de los objetivos establecidos para llevar acabo cual es el impacto que está obteniendo este nuevo sistema en el país.

Para determinar los diferentes criterios de la población acerca de los aspectos económicos que inciden de manera general en las transacciones que a diario realizan los habitantes como zona comercial que esta representa. Esto se realizó mediante la investigación formativa en la cual se dieron los procedimientos que esto implica.

El cuestionario diseñado para la recolección de datos mediante la encuesta está formado por 14 preguntas, las cuales distribuidas en dos secciones, la primera sección están relacionada con la información básica del informante y la segunda sobre las variables de estudio sobre información general del sistema de dinero electrónico en los procesos y las actividades económicas, considerado por: (Méndez Álvarez C. E., 2009).

\subsection{Procesamiento $\mathrm{y}$ análisis}

La información se procesó en función de los instrumentos y técnicas utilizadas, en la investigación de los perfiles de los consumidores de la Península de Santa Elena. En lo que se refiere a la tabulación, se utilizó el programa estadístico SPSS 10.1.

\section{Resultados}

\subsection{El dinero electrónico hoy en día se considera una herramienta que podría incidir en diferentes procesos}

El sistema de dinero electrónico incidirá en los siguientes procesos: incidiendo de manera positiva que se agilizaran las transacciones comerciales en un 54\%, siguiendo con un $30 \%$ que asegura que afectara en la 
comunidad y seguridad, mientras $9 \%$ fortalecerá plan del buen vivir vigente en nuestro país, además que un 4 $\%$ se alude que mejoraría el proceso de la economía familiar, mientras que un $3 \%$ opina que afectara en la vida cotidiana de las personas debido al cambio dentro del sistema monetario que adoptaran las personas, cuando entre en vigencia este sistema.

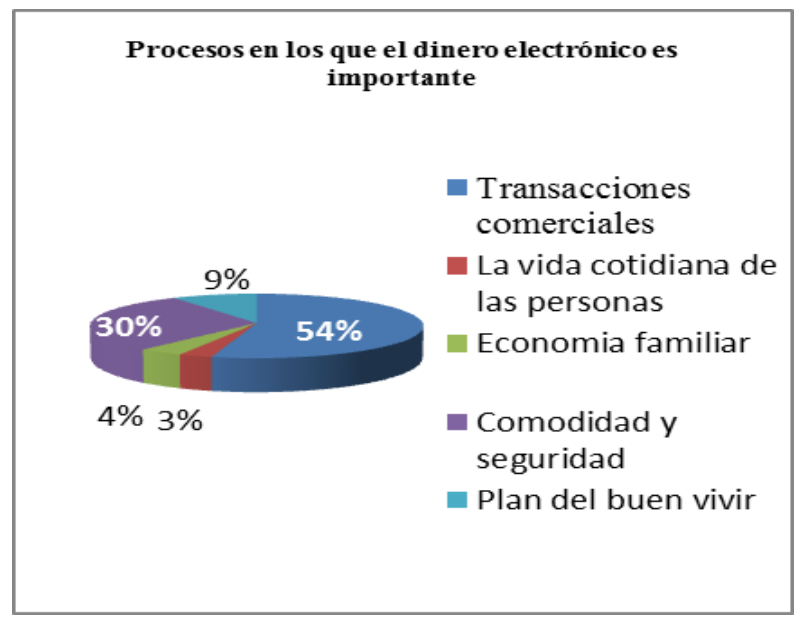

Figura No.- 1 Procesos en los que el dinero electrónico es importante

\subsection{Afectación del dinero electrónico a la economía del país.}

El $82 \%$ de encuestados mencionó, que cuando entre vigencia el nuevo sistema de dinero electrónico afectaría al país, debido a la falta de conocimiento, porque con este sistema el país se estaría ahorrando alrededor de 3 millones de dólares anuales por concepto del cambio de billetes por deterioro, y con un menor porcentaje del $18 \%$ que no afectaría porque se alude que el cambio es progresivo para la economía interna del país.

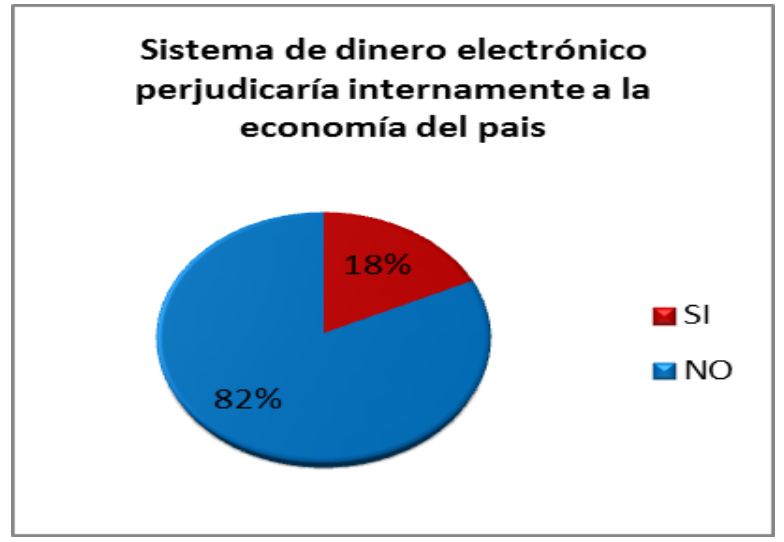

Figura No.- 2 Factores determinantes en proceso de decisión de compra
3.3 El conocimiento que tiene los comerciantes sobre la aplicación del sistema de dinero electrónico.

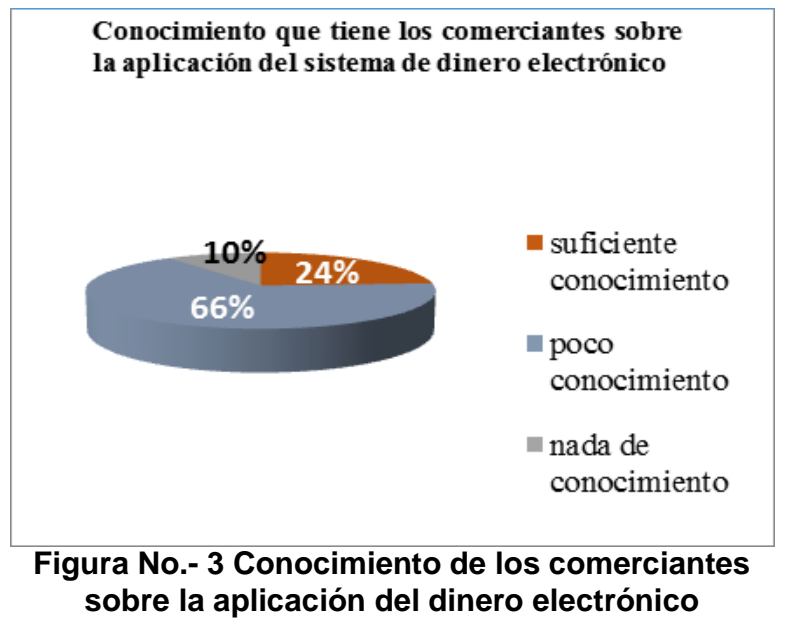

Se deduce que $66 \%$ los comerciantes poseen poco conocimiento acerca del dinero electrónico, mientras que el $24 \%$ tiene el conocimiento necesario para el manejo del sistema, sin embargo, el $10 \%$ de la población no cuenta con ningún tipo de información que sustente falencia.

\subsection{Necesidad e interes de capacitación de las personas sobre dinero electrónico}

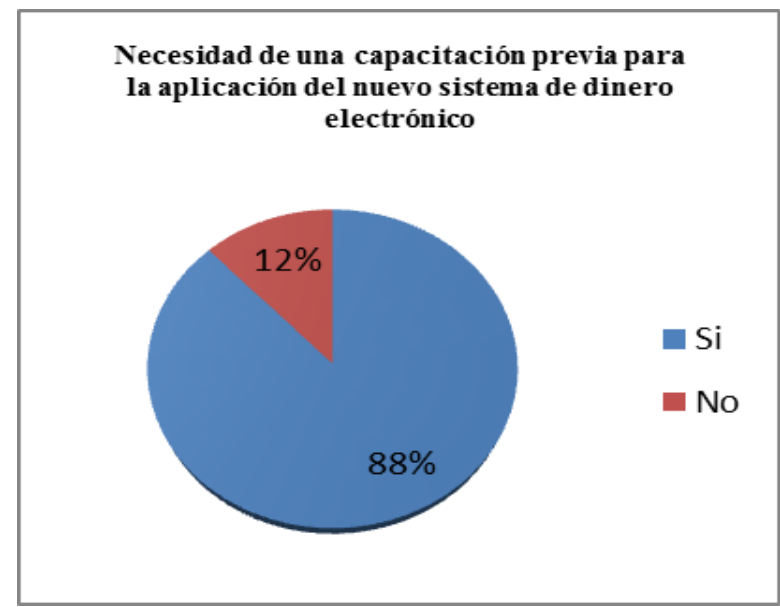

Figura No.-4 Necesidad de capacitación de dinero electrónico

De acuerdo a las estadísticas establecidas dentro del cantón La Libertad, respecto a la implementación del dinero electrónico se ha considerado que un $88 \%$ de la población necesita capacitación previa al uso del 
sistema, mientras que, un $12 \%$ concluyen que están lo suficientemente preparados

\section{5 ¿A través de que medio ha obtenido información sobre el sistema de dinero electrónico?}

Los medios de comunicación hoy se consideran una fuente de información esencial para la sociedad, ya que, provee un intercambio de ideas entre los usuarios que hacen uso de ellas, es por eso que en un $77 \%$ de la población escogida confirmo de este sistema por la televisión, seguido de del $11 \%$ que se informó por medio de internet y $6 \%$ en radio y diario.

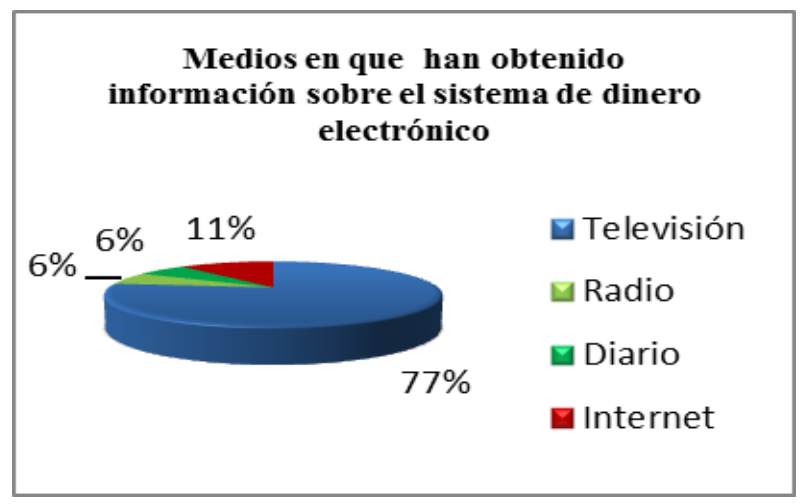

Figura No.-5 Medios en que han obtenido
información sobre el dinero electrónico

\subsection{Discusión}

Según Faz. X (2013). En la publicación realizada en el Portal de micro finanza con el tema "Nueva ola de dinero electrónico en América Latina" nos menciona lo siguiente: "la inclusión financiera no es un elemento nuevo para el sector bancario de la región".

El dinero electrónico es un tema revolucionario, que desde ciertas décadas está sujeto a cambios, la ola inicial la lideró a principios del presente siglo Brasil, donde, para llegar a más personas, los bancos adoptaron redes minoristas para que actuaran como agentes.

Según Faz. X (2013). En la publicación realizada en el Portal de micro finanza menciona: "Actualmente son tres los mercados que lideran el grupo de los que permiten a las entidades no bancarias emitir dinero electrónico: Bolivia, Perú y Brasil y otros en vía de desarrollo". Ya existen países que han preparado proyectos para esta actividad y se encuentran en fase de consultas: Colombia, El Salvador y Paraguay. México no parece contar con planes específicos para esta actividad, aunque sí tiene ciertas disposiciones en la legislación bancaria que, en la práctica, servirían para regular a los emisores no bancarios de dinero electrónico de forma similar a la regulación de que son objeto en otros países de América Latina y el Caribe. Parecen estar surgiendo tres formas distintas de regulación del dinero electrónico:

Desde entonces se han incorporado a la tendencia otros países como Perú, Colombia, México, y Ecuador generándose distintos modelos de servicio bancario a través de agentes, centrados unos en los pagos y otros en la descongestión de sucursales.

Según Martínez. D, (2014). En el artículo sobre el dinero electrónico publicado en el diario El Comercio menciona sobre las dudas que existe respecto a este sistema, señaló que "la persona que quiera disponer de dinero electrónico en su teléfono celular u otro dispositivo móvil deberá entregar el mismo valor en dólares americanos. Estos pasarán a formar parte de los activos del Central que permitirán cubrir el $100 \%$ del dinero electrónico solicitado", con la aportación del autor podemos exaltar que este sistema no significa la emisión de una nueva moneda, ni es un sustituto del dólar. Es un mecanismo de pago y cada dólar electrónico estará respaldado por un dólar físico y ayudara:

A los agentes económicos a realizar las transacciones comerciales de una manera eficiente y rápida, mediante un dispositivo móvil.

A los usuarios; porque se trata de un proyecto de inclusión sobre aquellas personas que no tienen acceso a banca móvil y mediante este sistema podrán participar de los diferentes tipos de transacción; cabe recalcar que antes de utilizarlos deberán estar previamente capacitados.

Otro de los beneficiarios de este proyecto será especialmente nuestro país, porque este sistema significará un ahorro de alrededor de 3 millones de dólares anuales para el Estado al disminuir el número de billetes que deberán cambiarse por deterioro.

La publicidad digital proporciona información importante sobre los productos, provocando que los consumidores compren vía on line o asistan a las tiendas físicas aun por curiosidad, en base a la percepción de la atención al cliente, calidad, características y precios de los productos determinan o no la compra. 
También produce un contacto directo con el público objetivo y muestra preocupación por el cliente tratando de lograr un alto nivel de satisfacción.

\section{Conclusiones}

- Es una renovación capitalista, que transformaría el comportamiento de las personas, pues la adopción del sistema monetario en el país se verían reflejadas en las transacciones comerciales.

- La existencia del sistema de dinero electrónico crea ventajas y desventajas que solo se perfeccionan con una buena regulación.

- La confianza que presentan los usuarios y los agentes económicos en este sistema de dinero electrónico traerá deliberación de obligaciones.

- En el ámbito de la capacitación existe la misión de que los comerciantes obtengan conocimientos, actitudes y habilidades en función de los objetivos del proyecto.

- El plan de capacitación es una actividad planificada cuya misión es incorporar el proceso del sistema de dinero electrónico que será dirigido en el país.

\section{Agradecimiento}

Mi más sincero agradecimiento al Ing. Jimmy Candell Soto, Rector de la muy ilustre Universidad Estatal Península de Santa Elena por su arduo trabajo y por gestionar el aporte de la comunidad universitaria en difusión de artículos formativos de gran interés; a todas las personas que colaboraron para que este trabajo saliera de la mejor manera posible.

\section{Referencias}

[1]Banco Central del Ecuador. Archivo No. 017-2011. El Directorio del Banco Central del Ecuador. Título XV Dinero Electrónico. Capítulo I, Articulo I.

[2]Banco Mundial 2012. Estadísticas. Países. Kenia.

[3]Bernal, C. A. (2010) Metodología de la investigación (Tercera Edición, ed.). (O. Fernández Palma, ed.) Bogotá Colombia: Person Education.
[4]Banco Central del Ecuador 2012. Estadísticas. Previsiones Macroeconómicas. Producto Interno Bruto.

[5]Regulación No. 055-2014 Del Banco Central del Ecuador. Quito, 28 de febrero de 2014. 